\title{
Down syndrome: The crucible for treating genomic imbalance
}

\author{
Julie R. Korenberg, $M D$, PhD
}

\begin{abstract}
History is merely a list of surprises... It can only prepare us to be surprised yet again. -Excerpt from Slapstick by Kurt Vonnegut
\end{abstract}

$\mathrm{V}$ enturing beyond the horizon is hazardous but such risks have expanded our universe and defined our world. Guided by our knowledge of human genome and human neurobiology, the Down syndrome (DS) field is expanding the familiar and gingerly exploring what steps must be taken to fundamentally change our approach to DS. In this issue, the article by Mégarbané $^{1}$ has placed the diagnosis and treatment of DS in a historical context and focused on the use of current mouse models to guide the development of treatments and on the need for collaborative groups to use standardized outcomes measures to test their efficacy in people with DS. The emphasis of Mégarbané ${ }^{1}$ is the history and celebration of partially trisoic mouse models of DS. The emphasis of this commentary is first on a celebration of human models, particularly those with partial and full Trisomy 21, and their use in defining the neural systems disturbed in DS. Such models are critical to narrowing the search for the genes that contribute to the disturbed prenatal brain wiring and postnatal brain function in DS. The second emphasis of this commentary is a set of provocative proposals intended to stimulate discussion and engender different ways of thinking about mouse models and human treatment trials for DS.

It was 1972 and human genetics was exploding. The first genomic causes of human disease had been mapped and unbalanced genomes were established as a cause of human disease. The genome had color and chromosomes had personalities, bands. In my second year of graduate school, humans finally loomed as appropriate experimental organisms with variations in chromosome architecture promising to bridge the secrets of the genome and their consequences for human development.

Trisomy for Chromosome 21 holds promise for understanding the genetic origins of a broad spectrum of human birth defects and adult disease. A major genetic cause of mental retardation and congenital heart disease, DS also confers the highest known genetic risk of duodenal stenosis, Hirschsprung disease, leukemia and Alzheimer disease, as well as several psychiatric disorders, including autism, anxiety, and depression. So much for the darker side of 21 in human disease. Trisomy 21 also has beneficial effects for its carriers, including lower heart

From the Center for Integrated Neuroscience and Human Behavior, The Brain Institute and Department of Pediatrics at the University of Utah, Salt Lake City, Utah.

Julie R. Korenberg, $\mathrm{PhD}$, MD, Center for Integrated Neuroscience and Human Behavior, The Brain Institute at the University of Utah, 383 Colorow Road, Room 362, Salt Lake City, UT 84108. E-mail: julie.korenberg@ hsc.utah.edu.

Disclosure: The author declares no conflict of interest

Submitted for publication July 13, 2009

Accepted for publication July 14, 2009.

Published online ahead of print August 17, 2009.

DOI: $10.1097 /$ GIM.0b013e3181b765e 7 rate and blood pressure and a lower risk of solid tumors. Surprisingly, none of the malformations, diseases, or beneficial conditions are clearly understood and their increased risk in Trisomy 21 is proof positive that there is a gene or genes on this smallest chromosome that, when only subtly overexpressed, disturb and identify disease pathways for human development as well as for major adult diseases, cardiovascular, cancer, mental illness, and dementia.

\section{Human systems for identifying genes and targeting treatment pathways for DS: Segmental trisomy}

Rare individuals with DS caused by duplications of only parts of Chromosome 21 provide the critical human models ${ }^{2-4}$ for narrowing the genes and focusing attention on the underlying mechanisms for malformations, neural, and cognitive features. Recently, this approach has been successful in implicating unsuspected genes for DS congenital heart disease ${ }^{4}$ and is a model for linking copy number variation to human disease. We will consider the qualities necessary and the ideal genetic features of such a panel for mapping human features. It is composed of individuals with aneuploidy only for Chromosome 21 and the number in the panel large enough to include uncommon phenotypes and a broad age range. Breakpoints must be measured with high resolution and fidelity and their distribution should span regions throughout the chromosome to produce duplications that evenly represent the entire chromosome. If all breakpoints were clustered, there would be no possibility to link smaller regions to specific phenotypes regardless of the number of cases. A broad spectrum of phenotypes can be mapped including subsets of cognitive performance and brain structure as well as physiology and biochemistry. However, the characteristics of a phenotype must be defined such that the risk ratio is high in DS versus in the normal population, because this strongly supports the inference that an overexpressed gene(s) on 21 promotes the development of the phenotype, e.g., the risk of atrioventricular septal defect in DS is $1000 \mathrm{X}$ versus normals. Ideally, most of the risk in the total population is accounted for by DS, e.g., 70\% of all atrioventricular septal defects are found in DS. This supports a major role of 21 gene(s) in altering a specific pathway rather than a pathway affected by innumerable genetic variations, and suggests that understanding the gene(s) in DS may elucidate previously unknown critical steps in human development.

Caveats to mapping include that when a phenotype requires more than one gene or cluster on Chromosome 21, only duplications that include both will confer a phenotype and mapping to the combined region is valid but no regions smaller than this can be defined. When a phenotype may be caused by any one of a number of genes (each of many genes can cause a phenotype) on Chromosome 21, small nonoverlapping duplications that include only one of them are needed to define the genes. Further, association of a given phenotype with a given chromosomal candidate region does not exclude the contributions of other regions. The larger the number of cases without the candidate region and without the phenotype, the more likely is 
the candidate region to be necessary and sufficient for the phenotype (for a formal Bayesian model, see Ref. 4). Finally, genes in chromosomal regions outside the candidate region may of course, contribute to modifications of the phenotype. Mapping phenotypes with segmental trisomies is a uniquely human and powerful approach when genetic and phenotypic constraints are met.

\section{Mapping genes for the neural systems and behavior disturbed in DS: The need for brain imaging}

"Intellectual disabilities" is an umbrella term and it is necessary to define the parts that are more specific to DS. This is an area that desperately needs research from multiple linked disciplines. Significant strides have been made recently ${ }^{5}$ to measure and parse cognitive performance and sleep behavior in DS and more work is needed for age-related comorbid psychiatric conditions. However, there is a great need to describe mappable features of brain structure and function in DS using emerging approaches for neuroimaging, high-resolution magnetic resonance imaging (MRI), functional MRI, diffusion tensor imaging, event-related potentials, and nuclear magnetic resonance spectroscopy. MRI is important for defining brain regions altered during development in DS and diffusion tensor imaging is important for defining brain tracts that underlie disturbed neural anatomic circuitry in DS. Functional MRI, event-related potentials and magnetoencephalography (MEG) can all probe brain function in response to cognitive stimuli and nuclear magnetic resonance spectroscopy can reflect neurotransmitter systems. ${ }^{6}$ For cognitive phenotypes, we need to compare data to parental means because much of the variation in the DS cognitive phenotype reflects the remainder of the genome, including genes that do not interact with those on Chromosome 21. Links to genes can emerge when research maps the neuroimaging and behavioral features in partial trisomies.

\section{A few provocative thoughts}

\section{Determining gene targets for drug therapy: Other uses for mouse models}

Although on a continuum with development, birth is the point after which DS is more readily treatable. One might divide the neural system and gene targets responsible for DS into those responsible for prenatal brain wiring and those responsible for postnatal functions. Mouse models would be ideal systems in which to determine the genes disturbing cognitive functions when overexpressed in an otherwise normally wired brain or that result in normal function when normally expressed in an otherwise trisomic brain.

What cognitive functions of the syndrome are due to the overexpression of genes and consequent disturbance of brain development before birth? That is, what would be the cognitive phenotype of a mouse that had DS throughout development but was corrected after birth? The answers to this define which systems are plastic and can be altered or ameliorated after birth. Those genes that disturb the development of a brain that nonetheless functions normally when corrected at birth would constitute clear targets for therapy.

What cognitive functions of DS are due to the overexpression of a given Chromosome 21 gene after birth? That is, if Chromosome 21 genes were overexpressed only after birth and not before, what cognitive functions would be abnormal? Some subsets of genes such as those involved in ongoing cellular functions or synaptic processes may affect both wiring and postnatal functions. Again, the question is which of the neural systems are plastic and what are the cellular processes that determine them. Those genes that disturb cognitive function in an otherwise normally wired brain would constitute clear targets for therapy.

Although speculative, these labor-intensive mouse models might be generated with large fragment models carrying tissue specific Cre-mediated deletion or inducible promoter systems acting at birth. ${ }^{7}$ The mouse cerebellum develops largely postnatally and, in addition to hippocampal specific tasks, it may provide a good system in which to test pre- versus postnatal effects of single genes.

\section{Caveats to mouse: Linking mouse to man}

Eighty million years have elapsed since a common ancestor, and cognition as well as brain size and structure are drastically different in mice versus humans. Therefore, despite their strengths and usefulness for pathogenesis and treatment models, it is not clear that some subtle aspects of cellular neuronal function and treatments developed in mice would apply in humans. It is important to link and verify findings in mice with those in humans. Previously a tremendous obstacle, this should now include studies from the metabolic and cellular, e.g., using mouse and human cell lines or stem cells, through the organismal, using neural imaging, cognitive testing, and human examples of mouse models, such as the partial trisomies for Chromosome 21. ${ }^{3,4}$ For example, although promising, there are no data implicating an abnormality in GABAergic tone in DS aside from the data from one mouse model, Ts65Dn mouse. Functional imaging in humans for the GABAergic system ${ }^{6}$ could address this. Mouse studies have an additional problem that many treatments do not transfer to humans. Making "humanized" mice with human Chromosome 21 fragments is a partial solution but the products of these human-controlled gene sets are still interacting within a genome expression control network that is $\sim 99 \%$ mouse.

\section{Treating DS with presumptive drugs targeted to basic neurotransmitter mechanisms: Humility in the face of human neurobiology}

What are the keys to understanding and treating DS and the plethora of associated diseases? For the goal of elucidating the mechanisms of human disease, studies of networks, single gene mechanisms, mouse and cellular models etc., are critical. However, it has been a justifiably frustrating experience to develop candidate drugs for DS based on our limited knowledge of human neurobiology (reviewed in Ref. 1) and even a good rationale is highly likely to be wrong. Is it time to take another tack? It is critical that we do no harm, but lack of action is not the antidote. To ameliorate the cognitive deficits, perhaps there are other, more direct routes that should be considered. This would bypass the need for understanding all of medical neurobiology and capitalize on what is known and already approved for the diploid population. Why not establish treatment cohorts, get the families and health systems engaged and test presumptively? Let us consider the following: Given that many would think it likely that there are ongoing postnatal/adult disturbances of brain function in DS some of which likely disturb synaptic function through alteration of the major neurotransmitter systems, why not define a series of approved medications to target the major neurotransmitter systems. In fact, many with DS are likely already treated with these drugs for various reasons.

The way forward would engage multidisciplinary teams with expertise in preclinical and clinical pharmacology of neural systems, pediatric and adult clinical trials, health systems eco- 
nomic outcomes, mouse behavior (particularly hippocampus), human genetics, and clinical medicine. There is a need for learning a new language and for tolerating naïve questions among experts. Goals would include determining systems and corresponding drugs to be targeted, evaluating the safety and potential adverse events of even approved drugs for indications in DS, considering the need for Investigational New Drug applications or for further study in animal models, the feasibility of Phase I trials, and in some cases designing the clinical trial. We as a field must get on with it, and in a reversal of the usual model, successes (or failures) in such presumptive clinical trials for treating cognitive deficits in DS could, in a reciprocal manner, then be followed by targeted studies of the mechanisms in cellular and animal models.

\section{Treat Chromosome 21: Turning down the third copy or its genes}

In 50 years perhaps, we will avoid the neurobiology entirely and treat the chromosome directly, a highly speculative model for the treatment of other copy number variations.

Regardless of theory, it is broadly agreed that DS is due to the imbalances of gene expression and disturbances of genomewide gene networks caused by the presence of a third Chromosome 21 . Some gene phenotypes may involve largely single genes or clusters on 21 and others may involve multiple genes on 21 , but the greatest effect of DS is on the complex and poorly understood networks of genes located in the other $98.3 \%$ of the genome. Treating or normalizing these networks is an important but daunting task. Simpler to imagine is turning off the expression of the third or turning down the transcription of all three copies of 21 or its genes. Approaches to this may include drugs with genome-wide effects such as histone deacetylase (HDAC) inhibitors ${ }^{8}$ that surprisingly are already emerging on the market (e.g., valproate). Alternatively, also very much in the experimental stage would be the use of RNA targeted to gene variations that are specific to one of the three copies. These experiments have all the challenges of drug generation and delivery but could be done both in cell or neuronal systems as well as in persons with DS. Although quite far off, one might begin to consider ways to turn down an entire 21 , perhaps using information that is likely to be gleaned in the next decade from understanding the mechanism of $\mathrm{X}$ inactivation, in which one of the two X's is turned off in each cell of normal females. It appears from rare individuals with X;21 translocations $^{9}$ that a subset of the genes on Chromosome 21 may be turned off by the same mechanisms regulating the decreased $\mathrm{X}$ expression in women. This approach could be developed in cellular systems. Challenges would include gene targeting, tissue access, drug delivery, among others but these are in common with treatments for neurodegeneration and cancer and are for the future.

\section{CONCLUSION}

Fifty years ago was the beginning, a genomic cause of DS that freed and focused thinking. What assumptions must now be cast aside? Even 15 years ago, it was not acceptable to talk of treating Trisomy 21, too many genes, too many interactions and too little known about neural systems, let alone their differences in DS. Today, it is not acceptable to talk of turning off or eliminating a chromosome. Perhaps, 50 or fewer years will see the treatment of DS and other aneuploidies as we now glimpse them, as genomic copy number variants whose treatment will echo the innocent and incisive questions of parents "why can't you just remove or turn off the extra 21?" Now, there is a challenge worthy of the best and the brightest of the next generation who must be seduced, charmed and enticed, turned on, stimulated, sparked to turn the field in another direction that is generalizable broadly to the treatment of genetic disorders . . . and most disorders are.

\section{ACKNOWLEDGMENTS}

JRK is USTAR Professor of Circuits of the Brain, and supported by Grants from the NIH (R01 HDO 45225; PO1 HD33113). I thank Charles J. Epstein for insightful comments.

\section{REFERENCES}

1. Mégarbané AR, Mircher C, Sturtz FG, Rethoré MO, Delabar JM, Mobley WC. The 50th anniversary of the discovery of trisomy 21: the past, present and future of research and treatment of Down syndrome. Genet Med 2009; 11:611-616.

2. Korenberg JR, Chen XN, Schipper R, et al. Down syndrome phenotypes: the consequences of chromosomal imbalance. Proc Natl Acad Sci U S A 1994; 91:4997-5001.

3. Lyle R, Bena F, Gagos S, et al. Genotype-phenotype correlations in Down syndrome identified by array CGH in 30 cases of partial trisomy and partial monosomy chromosome 21. Eur J Hum Genet 2009;17:454-466.

4. Korbel JO, Tirosh-Wagner T, Urban AE, et al. The genetic architecture of Down syndrome phenotypes revealed by high resolution analysis of human segmental trisomies. Proc Natl Acad Sci U S A 2009;106:12031-12036.

5. Nadel L, Allman M, Capone G, et al. Preliminary results from the development and validation of a neuropsychological battery for genetic studies in Down syndrome. Society for Neuroscience, Washington, D.C., November 15-19, 2008.

6. Jissendi Tchofo P, Baleriaux D. Brain (1)H-MR spectroscopy in clinical neuroimaging at 3T. J Neuroradiol 2009;36:24-40.

7. Nakamura S, Watanabe S, Ohtsuka M, et al. Cre-loxP system as a versatile tool for conferring increased levels of tissue-specific gene expression from a weak promoter. Mol Reprod Dev 2008;75:1085-1093.

8. Guan JS, Haggarty SJ, Giacometti E, et al. HDAC2 negatively regulates memory formation and synaptic plasticity. Nature 2009;459:55-60.

9. Couturier J, Dutrillaux B, Garber P, et al. Evidence for a correlation between late replication and autosomal gene inactivation in a familial translocation t(X;21). Hum Genet 1979;49:319-326. 